\title{
Impact of Changing Post-Operative Flexor Tendon Rehabilitation Program with Early Detection of Complications
}

\author{
KHALED A. REYAD, M.D.; NIVEEN F. AL-MAHMOUDY, M.D.* and HALA ABDELSABOUR, M.D.** \\ The Departments of Plastic, Burn and Maxillofacial Surgery* and Physical Medicine and Rehabilitation**, Faculty of Medicine, \\ Ain Shams University, Cairo, Egypt
}

\begin{abstract}
Background: Hand injuries are among the most frequent injuries worldwide, constituting between $6.65 \%$ to $28.6 \%$ of all injuries and $28 \%$ of injuries to the musculoskeletal system. Early post-operative rehabilitation and splinting after flexor tendon repair is very important to improve tendon healing. Presence of dedicated physical therapists play a golden role in early rehabilitation and diagnosis of tendon complications.

Patients and Methods: Data collected from all admitted cases with hand injuries during the period (from January 2016 to December 2017). In late 2016, a dedicated physical rehabilitation hand subunit was created in Physical Medicine and Rehabilitation Department. Hand ultrasound was done to exclude tendon rupture or tendon adhesions.

Results: 475 cases of flexor tendon injury, zone II injuries for fingers were the most common meanwhile, for thumb injuries; zone III in 2016 and zone II in 2017 were the most common, the rate of flexor tendon rupture in 2016 was $4.32 \%$ (3.3\% was early rupture within three weeks) while in 2017 was $2.87 \%$ (2.6\% were early rupture after three weeks), Tendon adhesions occurred in $7.32 \%$ of total cased in 2016 while in 2017 was $4.76 \%$.

Conclusion: Epidemiological data enhances our knowledge of injury patterns and may play a role in the treatment of tendon injuries. With early physical rehabilitation, better prognosis was achieved and hence better functional outcome.
\end{abstract}

Keywords: Flexor tendon injury - Rehabilitation.

\section{INTRODUCTION}

Hand injuries are among the most frequent injuries worldwide, constituting between $6.65 \%$ to $28.6 \%$ of all injuries and $28 \%$ of injuries to the musculoskeletal system [1]. Acute traumatic tendon injuries of the hand and wrist are commonly encountered in emergency rooms with incidence rate of 33.2 injuries per 100,000 people in mixed urban and rural Midwest country in the United States in 2014. The majority of cases involved a single tendon injury, with extensor tendon injuries occurring more frequently than flexor tendons [2].
Although outcomes after flexor tendon repair have been reportedly improving with modern treatments, complications are common with rates of re-operation of $6 \%$, rupture of $4 \%$, and adhesions of $4 \%$ [3]. Early post-operative rehabilitation and splinting after flexor tendon repair is very important to improve tendon healing, increase tensile strength, decrease adhesion formation, early return of function and less stiffness and deformity [4]. Presences of dedicated physical therapists play a golden role in early rehabilitation and diagnosis of tendon complications.

In this study, the role of early physical rehabilitation using the active controlled mobilization protocol was emphasized with early detection of flexor tendon complications using ultrasonography.

\section{PATIENTS AND METHODS}

This retrospective comparative study was conducted in Plastic Surgery Department; Ain Shams University Hospital. Data were collected from all admitted cases with hand injuries during the period (from January 2016 to December 2017) to document the frequency of hand injuries, specifically flexor tendon injuries. Single or multiple flexor tendon injuries were included. All demographic data of patients was documented including type of repair, post-operative rehabilitation, incidence of complications and recovery. Exclusion of crushing injuries with fractures, digital nerve injury, extensor tendon injuries or soft tissue defects; to avoid adhesions or delayed mobilization of tendon injuries.

All tendons were repaired using four strand core sutures by proline $2 / 0$ or $3 / 0$ according to the zone of injury to the tendon, followed by epitenon sutures by $5 / 0$ proline [5]. Dorsal blocking splint was applied to all patients. Early in this study (2016), the modified Kleinert's [6] early controlled 
motion protocol was used as post-operative rehabilitation program, where the wrist was supported with a dorsal blocking splint in $30^{\circ}$ of volar flexion, the meta-carpo-phalangeal joints in $60^{\circ}-70^{\circ}$ flexion and the inter-phalangeal joints straight. Rubber band traction was used for passive flexion. Four weeks post-operatively, a dorsal blocking splint was modified to hold the wrist in neutral position and was used only at night as a protective splint.

In late 2016, a dedicated physical rehabilitation hand subunit was created in Physical Medicine and Rehabilitation Department. The Pettengill [7] early mobilization post-operative rehabilitation program was used instead of modified Kleinert's [6] early controlled motion protocol. All patients were splinted in dorsal blocking splint with wrist in $20^{\circ}$ flexion, MCP joint in $70^{\circ}$ flexion and Interphalangeal joints (IP) in full extension. Rehabilitation program started within the first 3 days after tendon repair. The program passed through 4 phases. The criteria for advancement from one stage to another depend on flexion lag (discrepancy between active and passive flexion).

1- Phase I (first day to 3-4 weeks): Begin with passive PIP/DIP flexion in splint followed by active extension to the border of splint, then passive flexion to all finger joints followed by active extension to border of splint, then place and hold mobilization (which depend on placing the joint in flexion position by the therapist and ask the patient to maintain this position by his power).

2- Phase II (3-6 weeks): Continue as phase I with addition of place and hold hook fist tenodesis (flexion for PIP, DIP with extension MCP and wrist joint).

3- Phase III (6-8 weeks): Discontinue the dorsal block splint, may need extension ones. Active tenodesis progressed toward active tendon glides (which are repetition of hook fist, straight fist, full fist to straight hand in a quick and repetitive manner) for all hand Fig. (1).

4- Phase IV (8-16 weeks): Full active motion.

Comparison of the rate of tendon complications between years 2016 and 2017 showed the benefits of contribution of this subunit. Hand ultrasound had a good role in judging tendon rupture versus tendon adhesions, it was done if tendon rupture was suspected (evidenced by inability to flex interphalangeal joints, with no improvement in flexion lag with sessions) or if there was large difference between active and passive flexion movement (suspected partial tendon tear or adhesions) Fig. (2).

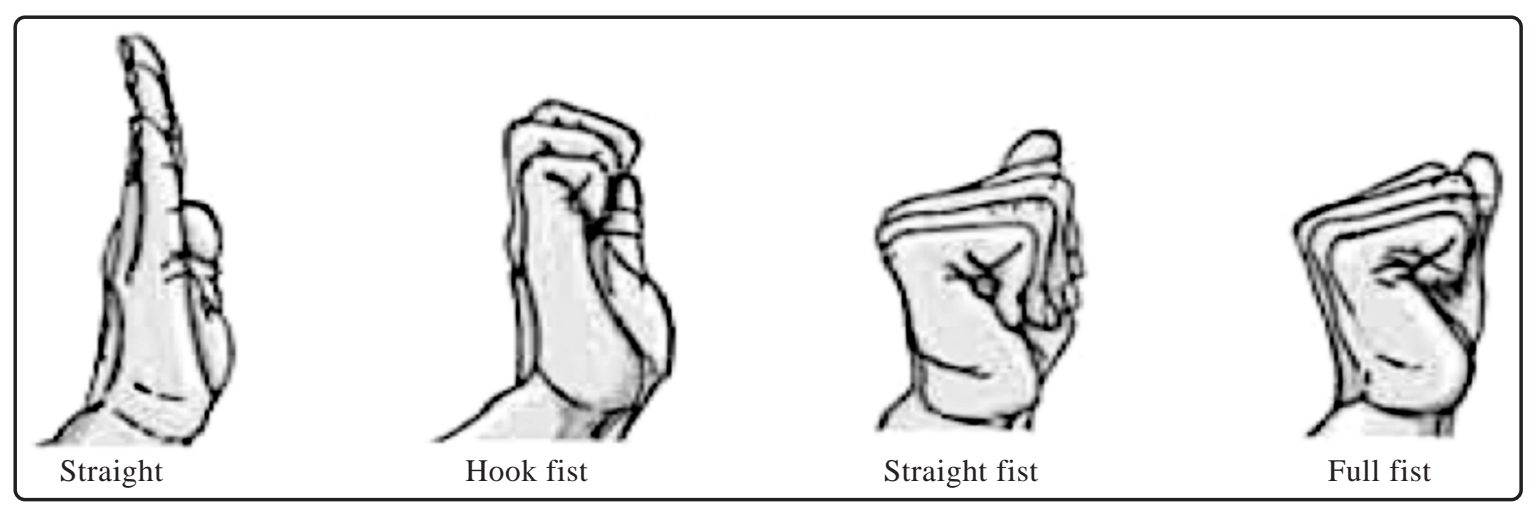

Fig. (1): Active tendon glides.

Fig. (2): (Left) Ultrasound image of normal flexor tendon (Right) Ultrasound image of rupture tendon (PP is proximal phalanx, MCP is metacarpal head).
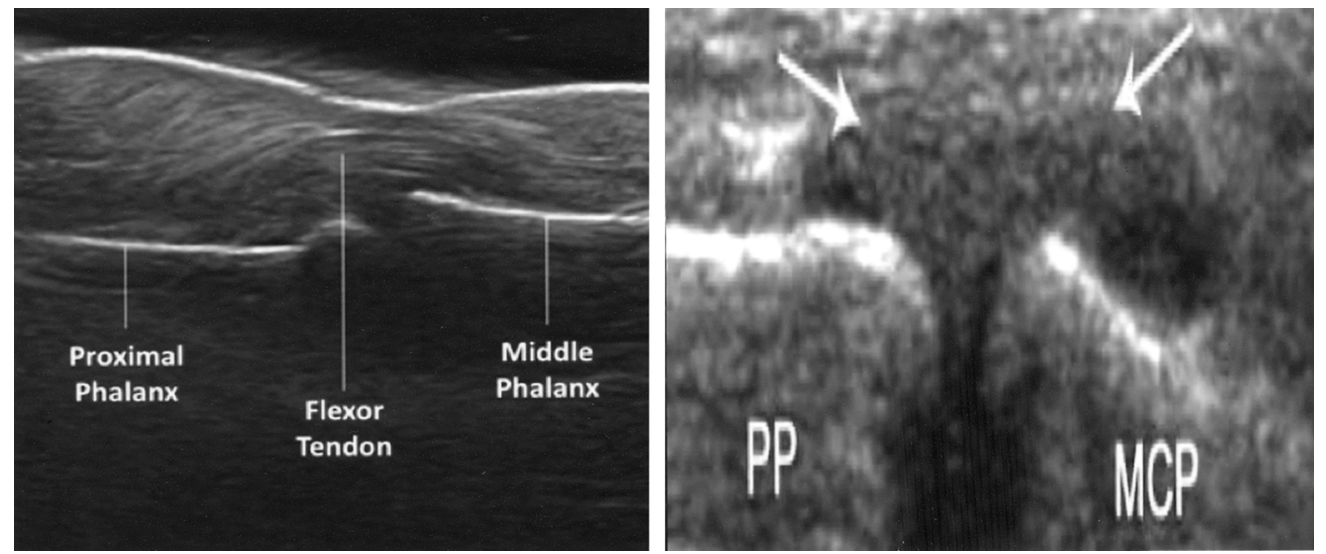
All patients were followed for at least 6 months post-operatively with complete recording of the complications as tendon rupture, adhesions and the rate of re-operations for tendons.

In case of limited active flexion; hand ultrasound was done to differentiate tendon rupture from adhesions. In case of tendon rupture, prompt interference is a must. In case of early rupture (within three weeks); trial of delayed primary repair is done. In case of delayed rupture (after three weeks) or failed delayed primary repair in early rupture, two stage tendon repair is done; where first stage is application of silicone rod (size 2$4 \mathrm{~mm}$ ) underneath flexor sheath pulleys, to create a gliding sheath for the tendon graft. Second stage is application of palmaris tendon graft (or plantaris if palmaris tendon is not available) in the track formed by the silicone rod. This was followed by the same rehabilitation program of the acute flexor tendon.

In case of tendon adhesions; failure to show improved active digital motion despite continued therapy implements tenolysis after serial joint measurements, where complete exposure of the whole tendon inside the tendon sheath and lysis of all the adhesions all around the tendons. This was followed by aggressive active rehabilitation which began the second day post-operatively.

\section{RESULTS}

In this study, 475 cases of flexor tendon injury were repaired early after trauma. The average age was 28.34 with the youngest age was 6 years and the oldest was 57 years, $87 \%$ were male and $13 \%$ were female.

The frequency of flexor tendons injuries in fingers and thumb were recorded in both years. The results showed that zone II injuries for fingers were the most common in the two years $35 \%$ in 2016 and $32 \%$ in 2017); meanwhile, for thumb injuries; zone III in 2016 (29\%) and zone II in 2017 (31\%) were the most common encountered injuries (Table 1). The rate of flexor tendon rupture in 2016 was $4.32 \%$ (3.3\% was early rupture within three weeks) while in 2017 was $2.87 \%$ (2.6\% were early rupture after three weeks). This dramatic and statistically significant decline in rate of tendon rupture is owed to further collaboration between plastic and physical therapy departments. Early ruptured was further evidenced by hand ultrasound and underwent either delayed primary repair (early rupture) or two stage tendon reconstruction (delayed rupture or failed delayed primary repair).
Tendon adhesions occurred in $7.32 \%$ of total cased in 2016 while in 2017 was $4.76 \%$. This dramatic and statistically significant decline in rate of tendon rupture is owed to further collaboration between Plastic and Physical Therapy Departments. In 2016, patients underwent tenolysis were $7.22 \%$ of total cases, $0.1 \%$ of total cases suffered tendon adhesions that were well managed by aggressive rehabilitation. In 2017 patients underwent tenolysis were $3.96 \%$ of total cases, $0.8 \%$ of total cases suffered tendon adhesions that were well managed by aggressive rehabilitation. The rate of complications is shown in Table (2).

Table (1): Frequency of tendon injuries during 2016 and 2017.

\begin{tabular}{|c|c|c|c|c|c|c|c|c|c|}
\hline \multirow{2}{*}{$\begin{array}{l}\text { Zone of } \\
\text { injury }\end{array}$} & \multicolumn{5}{|c|}{$\begin{array}{l}\text { Flexors of fingers } \\
\text { (zone of injury) }\end{array}$} & \multicolumn{4}{|c|}{ Flexor polises longus } \\
\hline & $\mathrm{Z} 1$ & $\mathrm{Z} 2$ & $\mathrm{Z} 3$ & $\mathrm{Z} 4$ & $\mathrm{Z} 5$ & $\mathrm{Z1}$ & $\mathrm{Z} 2$ & $\mathrm{Z} 3$ & $\mathrm{Z4}$ \\
\hline Year 2016 & $19 \%$ & $35 \%$ & $11 \%$ & $10 \%$ & $25 \%$ & $27 \%$ & $21 \%$ & $29 \%$ & $23 \%$ \\
\hline Year 2017 & $16 \%$ & $32 \%$ & $14 \%$ & $16 \%$ & $22 \%$ & $25 \%$ & $31 \%$ & $24 \%$ & $20 \%$ \\
\hline
\end{tabular}

Table (2): Incidence of tendon complications and rate of reoperation.

\begin{tabular}{lcccc}
\hline Year & $\begin{array}{c}\text { Total number } \\
\text { of cases }\end{array}$ & $\begin{array}{c}\text { Tendon } \\
\text { rupture }\end{array}$ & $\begin{array}{c}\text { Tendon } \\
\text { adhesions }\end{array}$ & $\begin{array}{c}\text { Overall } \\
\text { reoperation rate }\end{array}$ \\
\hline 2016 & 231 & $4.32 \%$ & $7.23 \%$ & $11.54 \%$ \\
2017 & 244 & $2.87 \%$ & $4.76 \%$ & $6.76 \%$ \\
\hline
\end{tabular}

\section{DISCUSSION}

The economic, social and physical impact of the disability that arises out of workplace hand injuries is tremendous. Hand trauma, often affects the younger population, is greater in the male sex and in the majority of cases is related to occupational, or assault injuries [4]. In our study hand trauma mainly affect younger age group especially third decade group, common in male due to occupation injuries more than female (household accidents), 475 cases had flexor tendon injury in the two years common in zone II; meanwhile, for thumb injuries; zone III in 2016 and zone II in 2017 were the most common encountered injuries.

Hand injury centers should be equipped with operating microscope and instruments with a dedicated physiotherapy and rehabilitation team [1]. The success of the surgical tendon repair depends very much on the application of physical therapy and splinting [4]. Physiotherapy must be systematic and constantly supervised by the hand surgeon with application of multi-modality approach [8]. 
In 2017, a new subunit hand team was established in Physical Medicine and Rehabilitation Department in Ain Shams University which was enthusiastic for hand injury rehabilitation. Thus with continuous cooperation with the Plastic Surgery Department, there was a decrease in the rate of complications reported in post-traumatic hand injuries when compared with the year 2016. A major complication was defined as either a rupture of a repaired tendon or adhesions [9]. These complications in most cases led to re-operation. Our re-operation rate was variable through-out the study it decreased from $11.54 \%$ in 2016 to $6.76 \%$ in 2017. This was accomplished with adequate tendon mobilization and early diagnosis of tendon complications thus early adequate intervention.

\section{Conclusion:}

Epidemiological data enhances our knowledge of injury patterns and may play a role in the treatment of tendon injuries. With early physical rehabilitation, better prognosis was achieved and hence better functional outcome with reducing lost work time and less economic burden.

It is important for surgeons and health care providers to understand the epidemiology and incidence of acute tendon injuries in the hand and wrist as even a relatively small injury may result in a significant clinical, social and economic impact on the patient and society.

\section{REFERENCES}

1- Prasad R., Bhamidi A., Rajeswaran A., Muthukumar S., Kothandaraman P. and Sivaraj M.: Epidemiology and Sequelae of Workplace Hand Injuries at a Tertiary Trauma Care Centre Surgical Science, 5: 150-8, 2014.

2- De Jong J.P., Nguyen J.T., Sonnema A.J.M., Nguyen E.C., Amadio P.C. and Moran S.L.: The Incidence of Acute Traumatic Tendon Injuries in the Hand and Wrist: A 10Year Population-based Study Clinics in Orthopedic Surgery, 6: 196-202, 2014.

3- Christopher J., soria A.H. and Timothy R.: Complications after flexor tendon repair: A systematic review and metaanalysis, J. of hand surgery, 37 (3): 543-51, 2012.

4- Rrecaj S.H., Martinaj M., Murtezani A., Dafina I.K., Haxhiu B. and Zatriqi V.: Physical therapy and splinting after flexor tendon repair in zone II Med. Arh., 68 (2): 128-31, 2014.

5- Strickland J.W.: Flexor tendon repair-Indiana method. The Indiana Hand Center Newsletter, 1: 1-19, 1993.

6- Edinburg M., Wid gerow A.D. and Biddulph S.L.: Early post-operative mobilization of flexor tendon injuries using a modification of the Kleinert technique J. Hand Surg., 12A: 34-8, 1987.

7- Pettengill K.M.: The evolution of early mobilization of the repaired flexor tendon, J. Hand Ther., 18: 157-68, 2005.

8- Hill C., Riaz M., Mozzam A. and Brennen M.D.: A Regional Audit of Hand and Wrist Injuries. A Study of 4873 Injuries. Journal of Hand Surgery, 23: 169-200, 1998.

9- Manninen M., Karjalainen T., Määttä J. and Flinkkilä T.: Epidemiology of flexor Tendon injuries of The hand in a northern Finnish population, Scandinavian Journal of Surgery, 106 (3): 278-82, 2017. 